\title{
Cdc13 both positively and negatively regulates telomere replication
}

\author{
Abha Chandra, ${ }^{1}$ Timothy R. Hughes, ${ }^{2,3}$ Constance I. Nugent, ${ }^{1,4}$ and Victoria Lundblad ${ }^{1,2,5}$ \\ ${ }^{1}$ Department of Molecular and Human Genetics and ${ }^{2}$ Program in Cellular and Molecular Biology, Baylor College \\ of Medicine, Houston, Texas 77030, USA
}

Cdc13 is a single-strand telomeric DNA-binding protein that positively regulates yeast telomere replication by recruiting telomerase to chromosome termini through a site on Cdc13 that is eliminated by the $c d c 13-2$ mutation. Here we show that $\mathrm{Cdc13}$ has a separate role in negative regulation of telomere replication, based on analysis of a new mutation, $c d c 13-5$. Loss of this second regulatory activity results in extensive elongation of the $\mathrm{G}$ strand of the telomere by telomerase, accompanied by a reduced ability to coordinate synthesis of the C strand. Both the $c d c 13-5$ mutation and DNA polymerase $\alpha$ mutations (which also exhibit elongated telomeres) are suppressed by increased expression of the Cdc13-interacting protein Stn1, indicating that Stn1 coordinates action of the lagging strand replication complex with the regulatory activity of CDC13. However, the association between $\mathrm{Cdc13}$ and $\mathrm{Stn} 1$ is abolished by $c d c 13-2$, the same mutation that eliminates the interaction between Cdc13 and telomerase. We propose that Cdc13 participates in two regulatory steps-first positive, then negative-as a result of successive binding of telomerase and the negative regulator Stn1 to overlapping sites on Cdc13. Thus, Cdc13 coordinates synthesis of both strands of the telomere by first recruiting telomerase and subsequently limiting G-strand synthesis by telomerase in response to C-strand replication.

[Key Words: Telomere; telomerase; Cdc13; Stn1; DNA replication]

Received October 20, 2000; revised version accepted December 27, 2000.

Telomeres are guanine-rich, simple repeat sequences that constitute the physical ends of eukaryotic chromosomes. The sequence and structure of these specialized DNA termini permits extension of the G strand by the enzyme telomerase, thereby counteracting sequence loss due to incomplete replication or degradative activities (Lingner et al. 1995; Greider 1996). In yeast and human cells, a telomerase deficiency leads to a progressive decline in telomere length that inhibits the proliferative capacity of the cell and heralds replicative senescence (Lundblad and Szostak 1989; Bodnar et al. 1998). Analysis of telomerase-deficient mice has also revealed an essential role for telomere maintenance in the long-term viability of high-renewal organ systems (Lee et al. 1998). Furthermore, loss of telomerase in the absence of checkpoint function can also promote genetic instabilities and carcinogenesis, especially in highly regenerative tissues (Chin et al. 1999; Artandi et al. 2000), illustrating the importance of telomeres in maintaining genomic stability.

Increasing attention has been directed at the importance of coordinating G-strand synthesis by telomerase

Present addresses: ${ }^{3}$ Rosetta Inpharmatics, Kirkland, WA 98034, USA. ${ }^{4}$ Department of Cell Biology and Neuroscience, University of California, Riverside, CA 92521, USA.

${ }^{5}$ Corresponding author.

E-MAIL lundblad@bcm.tmc.edu; FAX (713) 798-5931.

Article and publication are at www.genesdev.org/cgi/doi/10.1101/ gad.861001. with replication of the $\mathrm{C}$ strand of the telomere. Following extension of the $3^{\prime}$ terminus of the G-rich strand by telomerase, fill-in synthesis of the other strand, presumably by the machinery that normally performs lagging strand DNA replication, is thought to be necessary to prevent elongated single-stranded regions at chromosome termini. Several recent studies indicate that disruption of C-strand DNA synthesis can impair telomere length control (for review, see Price 1997; Evans and Lundblad 2000). In the ciliate Euplotes, inhibition of DNA polymerases $\alpha$ and $\delta$ during de novo telomere synthesis alters the length of the telomeric $\mathrm{C}$ strand and also causes an increase in the length and heterogeneity of the G strand (Fan and Price 1997). Similarly, propagation of $S$. cerevisiae strains defective for components of the lagging strand DNA replication machinery cause a telomerase-dependent increase in telomere length /Carson and Hartwell 1985; Adams and Holm 1996; Adams Martin et al. 2000). In addition, de novo telomere formation at a newly created double-strand break requires not only telomerase but also DNA polymerase $\alpha$ and $\delta$ (Diede and Gottschling 1999). Collectively, these results indicate that in both yeast and ciliates, G-strand and C-strand synthesis are tightly coregulated, although the molecular mechanism for this coordination has not been elucidated.

In yeast, a critical contributor to several aspects of telomere function is the protein Cdc13, which exhibits 
high affinity sequence-specific binding for single-stranded telomeric DNA (Lin and Zakian 1996; Nugent et al. 1996; Hughes et al. 2000a). Consistent with this substrate specificity, Cdc13 is associated with the telomere during S phase (C.I. Nugent and V. Lundblad, in prep.), which correlates with the transient increased singlestrand G-rich extension observed at yeast telomeres (Wellinger et al. 1993, 1996). CDC13 performs an essential activity necessary to protect chromosome termini, as loss of CDC13 function is accompanied by immediate and extensive loss of one strand of the telomere (Garvik et al. 1995; Diede and Gottschling 1999). In addition to this end protection function, $C D C 13$ positively regulates telomere replication by recruiting telomerase to the telomere, an activity that is abolished by the cdc13-2 missense mutation. The $c d c 13-2$ strain, which is normal for telomeric end protection, exhibits a telomere replication defect similar to that of a strain that lacks telomerase (Nugent et al. 1996), although telomerase activity in cellfree $c d c 13-2$ extracts is unaffected (Lingner et al. 1997). This defect is partially suppressed by increased expression of the telomerase-associated Est1 protein (Nugent et al. 1996), and a complex containing both proteins can be coimmunoprecipitated in yeast, using overexpressed recombinant versions of Est1 and Cdc13 (Qi and Zakian 2000). Furthermore, the cdc13-2 mutation can be reciprocally suppressed by a specific missense mutation in EST1 (Pennock et al. 2001), thereby demonstrating that positive regulation of telomere replication by $\mathrm{Cdc} 13$ is due to a direct interaction between $\mathrm{Cdc} 13$ and a subunit of telomerase, resulting in recruitment of the enzyme to the telomere.

In addition to this positive regulatory role, $\mathrm{Cdc} 13$ has been implicated in negative control of telomere length. The thermolabile $c d c 13-1$ mutant exhibits greatly elongated telomeres when grown at intermediate temperatures (Grandin et al. 1997). However, this mutant strain is also severely impaired for the essential end protection function of CDC13 (Garvik et al. 1995; Diede and Gottschling 1999); therefore, analysis of the $c d c 13-1 \mathrm{mu}-$ tation has not clarified whether the proposed role in negative length control is distinct from the essential function of $C D C 13$. An additional factor that contributes to negative length regulation is the Cdc13-interacting protein Stn1. These two proteins associate in a twohybrid assay and display a number of genetic interactions, suggesting that Stn 1 and Cdc13 function together at the telomere as a complex (Grandin et al. 1997). Furthermore, a mutation in STN1 increases telomere length by $\sim 1 \mathrm{~kb}$, leading Charbonneau and colleagues to propose that Stn1 is a negative regulator of telomere length (Grandin et al. 1997, 2000). Cdc13 is also associated with DNA polymerase $\alpha$, although mutations that disrupt this association have only a modest (50-150 bp) increase in telomere length (Qi and Zakian 2000); therefore, the contribution of the Cdc13-Pol $\alpha$ association to telomere length control is unclear.

We describe here the identification of a mutation of CDC13, cdc13-5, that exhibits a striking defect in negative regulation of telomere length, with no impact on the essential function of $C D C 13$. In this strain, telomere length is increased by $\geq 1000 \mathrm{bp}$ as a result of unregulated elongation of the G-rich strand by telomerase. This is accompanied by a reduced ability to coordinate synthesis of the C-rich strand, resulting in chromosome termini with greatly extended single-strand extensions of the G strand. The $c d c 13-5$ defect is dependent on the ability to recruit telomerase to the telomere, as cdc135 -mediated telomere elongation is blocked by the cdc13-2 mutation. Genetic interactions between the cdc13-5 mutation and DNA polymerase $\alpha$ also implicate misregulation of C-strand synthesis in this mutant phenotype. Therefore, $c d c 13-5$ defines a role for $\mathrm{Cdc13}$ in coordination of the synthesis of the two strands of the telomere, which is distinct from the telomerase recruitment activity defined by the cdc13-2 mutation. We propose that these two regulatory activities of Cdc13 correspond to two distinct steps in telomere replication that coordinate and regulate synthesis of the two strands of the telomere. In the first step, Cdc13 initiates telomere replication by recruiting telomerase, which extends the $3^{\prime}$ terminus of the G strand of the telomere. In the second step, fill-in synthesis of the $\mathrm{C}$ strand by lagging strand synthesis machinery acts to limit extension of the G strand by telomerase, an inhibitory activity that is defective in the $c d c 13-5$ mutant. We further suggest that these two steps are the consequence of successive binding of the telomerase-associated Estl subunit and the negative regulator Stn 1 to overlapping binding sites on Cdc13. By relying on a single protein as the regulatory center, this proposed mechanism provides a simple means for coordinating synthesis of the two strands of the telomere.

\section{Results}

\section{Cdc13 is a negative regulator of telomerase}

As part of our investigation of the Cdc13 protein, we uncovered two alleles of CDC13, cdc13-3 and cdc13-5, that result in a pronounced defect in telomere length maintenance. The telomeric tract, which is normally $\sim 350$ bp in a wild-type strain, was elongated by $\sim 500-$ 1000 bp in $c d c 13-5$ and $c d c 13-3$ strains, along with an accompanying increase in telomere length heterogeneity (Fig. 1A). The cdc13-3 mutation was the result of an alanine scan mutagenesis of CDC13 (Hughes 1998) and mapped to residues 695-699 at the C-terminal boundary of the Cdc13 DNA-binding domain. Western analysis, using an N-terminally epitope-tagged version (HA3Cdc13-3), indicated that the mutant Cdc13-3 protein apparently existed in two forms in yeast: the full-length $105-\mathrm{kD}$ protein and a truncated version that migrated at $\sim 80 \mathrm{kD}$, with both variants present in substantially reduced levels relative to the wild-type full-length Cdc13 protein (data not shown). We surmised that the $c d c 13-3$ mutation resulted in an unstable protein that was readily proteolyzed to remove $\sim 20 \mathrm{kD}$ from the C-terminal region of the protein. To test directly whether removal of the $\mathrm{C}$ terminus of $\mathrm{Cdc} 13$ was responsible for telomere elongation, an additional allele, cdc13-5, was con- 
Figure 1. $\mathrm{Cdc13}$ is a negative regulator of telomere elongation. (A) Telomere length of $C^{2} C_{13}{ }^{+}$(lane 1), cdc13-3 (lane 2), and cdc13-5 (lane 3). The cdc13-3 and cdc13-5 haploid strains were derived from DVL233 and DVL326, respectively, by tetrad dissection; DNA was prepared after $\sim 35$ generations of growth. (B) Anti-HA immunoblot of anti-HA immunoprecipitates from equal amounts of whole cell extract prepared from a $c d c 13-\Delta$ strain expressing HA3-Cdc13 (lane 1; pVL841), HA3-Cdc13-5 (lane 2; pVL903) and untagged Cdc13 (lane 3; pVL440); all plasmids were single-copy $C E N$ vectors, with CDC13 expressed from its native promoter. Arrowheads indicate the full-length Cdc13 protein $(\sim 116 \mathrm{kD})$ and the truncated Cdc13-5 protein $(\sim 80 \mathrm{kD})$. (C) Telomere length of CDC13/cdc13-5 EST1/est1-A RAD52/ rad52- $\Delta$ (lane 1), $C D C 13^{+}$(lane 2), rad52- $\Delta$ (lane 3), cdc13-5 (lanes 4,5), cdc13-5 rad52- $\Delta$ (lanes 6,7), est1- $\Delta$ (lane 8), and cdc13-5 est1- $\Delta$ (lane 9). The haploid strains shown in lanes

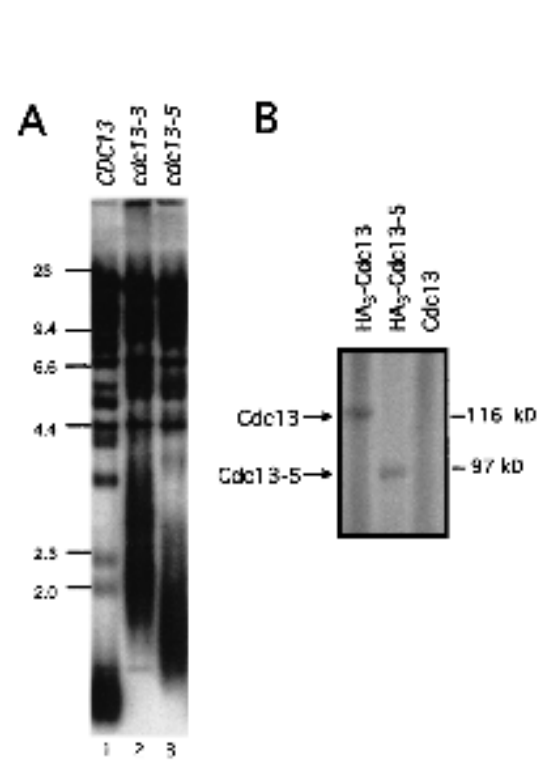

2-9 were obtained from DVL328 (lane 1) by tetrad dissection; DNA was prepared after $\sim 35$ generations of growth, except for lanes 5 and 7, which were grown for an additional 10 generations. (D) Telomere length of $c d c 13-5$ (lane 1), cdc13-2,5 (lane 2), cdc13-2 (lane 3), and $C D C 13^{+}$(lane 5); strains were generated by transforming $c d c 13-\Delta /$ pVL438 with pVL1033 (cdc13-5), pVL1360 (cdc13-2,5), pVL690 (cdc13-2), and pVL440 (CDC13), followed by eviction of pVL438 on 5-FOA. Although a cdc13-2,5 strain exhibits a senescence phenotype characteristic of a telomere replication defect, telomere length of the $c d c 13-2,5$ strain is reproducibly not quite as short as the cdc13-2 strain at any given time point (cf. lanes 2 and 3). This may be caused by residual recruitment activity of the Cdc13-2 mutant protein (which is consistent with the prior demonstration that the senescence phenotype of a cdc13-2 strain is somewhat delayed relative to telomerase null mutations; Lendvay et al. 1996), thereby allowing partial manifestation of the $c d c 13-5$ defect.

structed by the introduction of a stop codon following amino acid 694, which truncated the Cdc13 protein by 230 amino acids. This truncation occurs at the boundary of the DNA-binding domain, which can be expressed separately as a stable polypeptide (Hughes et al. 2000a). The cdc13-5 mutant yeast strain exhibited extensive telomere elongation to a degree roughly comparable to that observed with the $c d c 13-3$ strain (Fig. 1A). As described in more detail later in this article, the $c d c 13-5$ strain also grew as well as a wild-type strain. The Cdc13-5 protein was also produced at levels comparable to wildtype protein (Fig. 1B) in contrast to the situation with the cdc13-3 mutant strain. Therefore, unless otherwise noted, the experiments described in this article were conducted with the $c d c 13-5$ allele.

To determine whether the increase in telomere length was mediated by telomerase or recombination, telomerase-defective and recombination-defective $c d c 13-5$ double mutant strains were examined. In a $c d c 13-5$ strain that lacked telomerase (cdc13-5 t1c1- $\Delta$ or cdc13-5 est1- $\Delta$ ), telomere elongation was abolished, and the double mutant strain showed the senescence phenotype characteristic of telomerase null strains (Fig. 1C; data not shown). In contrast, telomere length in the $c d c 13-5$ strain was unaffected by loss of the major pathway for homologous recombination, as elongation occurred to the same degree in $c d c 13-5$ rad52- $\Delta$ and cdc13-5 RAD52 strains (Fig. 1C). Therefore, the greatly elongated telomeres observed in the $c d c 13-5$ strain are the result of action by telomerase. The $c d c 13-5$ mutation was recessive to $C D C 13^{+}$, indicating that the ability to negatively regulate telomere length is a property of the wild-type protein (Fig. 1C).

CDC13 also has a positive role in maintaining telomere length, which is eliminated by the $c d c 13-2$ mutation (Nugent et al. 1996). One model consistent with both positive and negative regulatory roles for $C D C 13$ proposes that the wild-type $\mathrm{Cdc} 13$ protein first recruits telomerase (an activity that is lost in the cdc13-2 mu$\operatorname{tant}$ ) and then subsequently acts to limit the degree of extension of the G-rich strand by telomerase (an activity that is lost in the $c d c 13-5$ mutant). A prediction of this model is that an allele of $C D C 13$ that contains both mutations should fail to recruit telomerase and, therefore, should not result in elongated telomeres. Consistent with this prediction, a $c d c 13-2,5$ strain, with both regulatory mutations introduced into the $C D C 13$ gene, no longer exhibited long telomeres. Instead, telomeres were short (Fig. 1D), and the $c d c 13-2,5$ strain showed a senescence phenotype (data not shown). Therefore, the loss of regulation of telomere length exhibited by the $c d c 13-5$ mutation depends on the telomerase recruitment function of $C D C 13$.

The cdc13-5 mutation results in greatly increased single-strand extension of the G-rich strand of the telomere

One means by which telomerase synthesis could become unregulated could be because of a loss of coordination 
between G-strand and C-strand synthesis. Impairment of DNA polymerase $\alpha$ results in elongated telomeres, as well as an increase in the extent of the terminal singlestrand extension of the G-rich strand /Carson and Hartwell 1985; Adams and Holm 1996; Fan and Price 1997; Adams Martin et al. 2000). To examine whether the cdc13-5 mutant similarly affected the extent of single-stranded G-rich DNA, nondenaturing in-gel hybridization was used to examine chromosome termini. In wild-type yeast, the G-rich strand can only be readily detected as single stranded in late $S$ phase, whereas at other points in the cell cycle, the chromosome terminus is either blunt or the overhang is below the detection limit (<30 bases; Wellinger et al. 1993). Therefore, due to both detection limits and the transient nature of the single strandedness of the $G$ strand during $S$ phase, single-stranded yeast termini cannot be reliably detected in asynchronous cultures of wild-type cells. However, when unsynchronized cultures of both cdc13-3 and cdc13-5 mutant strains were examined, G-rich singlestrand telomeric signals were readily detected using a strand-specific probe (Fig. 2A). Treatment with Escherichia coli ExoI, a $3^{\prime} \rightarrow 5^{\prime}$ single-strand exonuclease, abolished the signal, arguing for the presence of a terminal single-stranded G-rich overhang, as opposed to internal gaps or nicks. Moreover, no single strandedness corresponding to the C-rich strand was detected (data not shown). Strand separation gels that measured the length of the C strand showed that this strand was also elongated in a $c d c 13-5$ strain, relative to the length of the $\mathrm{C}$ strand in a wild-type strain (data not shown). Therefore, the increased single strandedness of the $G$ strand does not appear to be caused by extensive resection of the $C$ strand. These results indicate that increased elongation of the G strand by telomerase in this mutant strain is accompanied by a loss in coordination of the synthesis of the $\mathrm{C}$ strand.

The elongated terminal extensions detected in a cdc13-5 strain could reflect a defect during $S$ phase, when telomere synthesis by telomerase occurs (Marcand et al. 2000). The alternative possibility is that the altered $\mathrm{G}$ tails in the $c d c 13-5$ mutant are the consequence of an alteration in end structure that persists throughout the cell cycle, such as that observed in yku70- and yku80 mutants (Gravel et al. 1998; Polotnianka et al. 1998), possibly as a consequence of exposure of telomeres to unregulated activities other than telomerase. To distinguish between these two possibilities, we determined whether extensive elongation of the G-rich strand could be detected at different periods of the cell cycle, using synchronized cultures of the cdc13-5 mutant strain. Cells were arrested with $\alpha$ factor in $\mathrm{G}_{1}$ and subsequently released, and the G-rich strand of the telomere was examined on native gels using a strand-specific probe (Fig. $2 B)$. When cells were arrested in $\mathrm{G}_{1}$, no telomeric single strandedness could be detected. However, 40-60 min after release, when FACS analysis indicated that the majority of cells had entered S phase, the $c d c 13-5$ mutant exhibited a peak of single-stranded G-rich telomeric DNA. This signal declined as the cells exited $S$ phase and increased again during the next cell cycle, although the peak of the signal became less pronounced as the culture became more asynchronous. These results indicate that the single-stranded $\mathrm{G}$ tail is a substrate for extended synthesis by telomerase during S phase, resulting in the telomere length defect observed in the cdc13-5 mutant strain.

Overexpression of STN1 suppresses cdc13-5 and pol $\alpha$ telomere length defects

The similarity in phenotypes of the $c d c 13-5$ and DNA polymerase $\alpha$ mutant strains suggests that the same step in telomere replication may be affected, which is also consistent with the recent demonstration that Cdc13 and DNA Pol $\alpha$ can be coimmunoprecipitated /Qi and Zakian 2000). To provide further support for a functional relationship between these two proteins, we asked whether alterations in the level of the Cdc13-interacting protein, Stn1, could influence the phenotypes of either
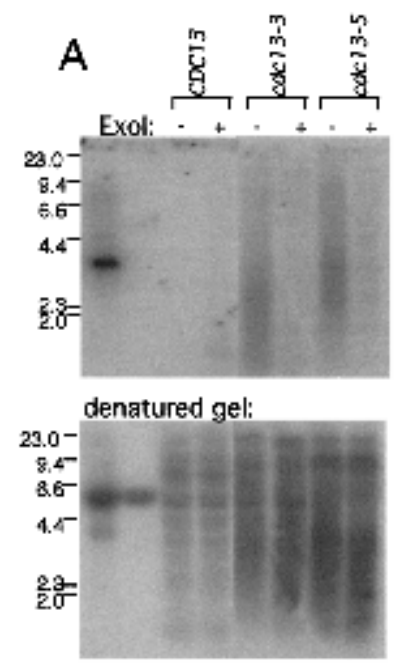

B

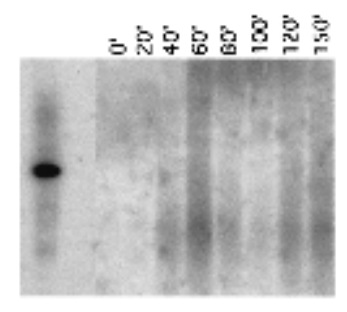

denatured gel:

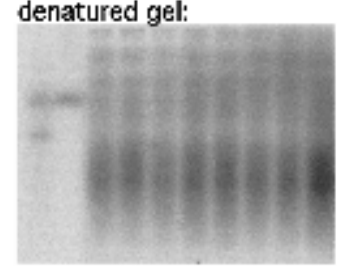

Figure 2. The $c d c 13-5$ strain exhibits an altered end structure during $S$ phase. (A) DNA from $C D C 13^{+}, c d c 13-3$, and $c d c 13-5$ strains were mock treated $($ lanes $3,5,7)$ or treated with ExoI (lanes 4,6,8), digested with XhoI, and analyzed by nondenaturing in-gel hybridization with a $\mathrm{C}_{1-3} \mathrm{~A}$ oligonucleotide probe (top); the same gel, denatured and rehybridized with the same oligonucleotide probe (bottom); lanes 1,2: pVL260 (with 80 bp of $\mathrm{G}_{1-3} \mathrm{~T}$ telomeric repeat DNA) heat denatured or nondenatured, respectively. Size markers: $\lambda$ HindIII-digested DNA. (B) DNA from a $c d c 13-5$ strain, prepared from time points collected every 20 min after release from an $\alpha$ factor-induced $\mathrm{G}_{1}$ arrest, was examined by native in-gel hybridization with a $\mathrm{C}_{1-3} \mathrm{~A}$ oligonucleotide probe (top, left), denaturation and rehybridization with the same probe (bottom, left), and FACS analysis (right); lanes 1,2: pVL260, denatured or nondenatured, respectively. 
pol1 or $c d c 13-5$ mutants. Notably, increased expression of STN1 suppressed the telomere phenotypes of mutations in both genes, implicating the $c d c 13-5$ defect in a step in telomere replication that depends on DNA polymerase $\alpha$ function. STN1 expression, driven by the GAL promoter and on a high-copy plasmid, reduced telomere length in a cdc13-5 strain to nearly wild-type length (Fig. 3A) whereas overexpression of STN1 had little or no effect on telomere length in a wild-type strain. In addition, single-stranded G-rich DNA was not detectable in asynchronous cultures of a cdc13-5 strain overexpressing STN1 (Fig. 3B), as would be predicted if the alterations in telomere length and the terminal G strand overhang are the result of the same defect. Increased expression of STN1 similarly influenced the telomere replication defect of DNA polymerase $\alpha$ mutants. The telomere elongation phenotype observed when pol1-12, pol1-16, and pol1-17 mutant strains were grown at semipermissive temperatures was substantially suppressed when STN1 was overexpressed (Fig. 3C; data not shown). Suppression was specific to the telomere replication phenotype of the pol1 mutant strains, as overexpression of STN1 did not suppress the temperature sensitivity of these pol1 mutant alleles (data not shown).

\section{Duplex telomeric chromatin is not altered in the cdc13-5 strain}

One mechanism for telomere length control proposes that telomeric chromatin equilibrates between an open conformation that permits telomerase access and a higher-order closed conformation that inhibits access of the telomerase enzyme through sequestration of the chromosome terminus (for review, see Evans and Lundblad 2000). This length-determining conformational switch is determined by binding of negative regulatory proteins to duplex telomeric DNA. Therefore, the increased telomere length of pol1 strains could be due to reduced assembly of inhibitory proteins on duplex telomeric DNA, possibly as a consequence of altered rates of

\section{A}

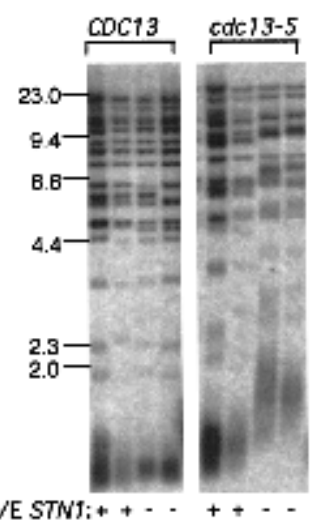

B

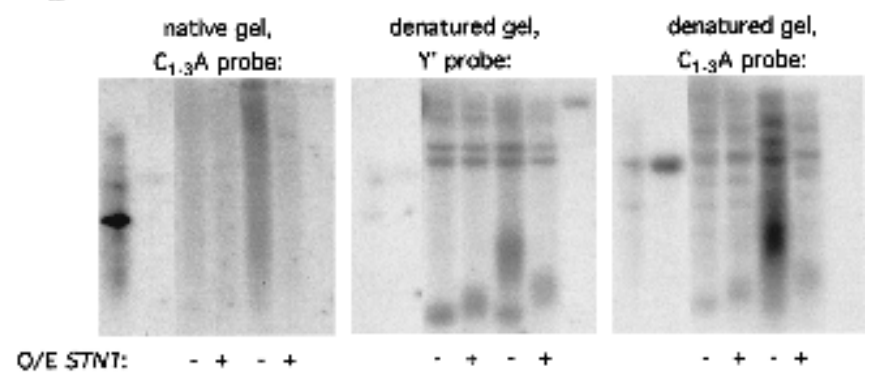

C

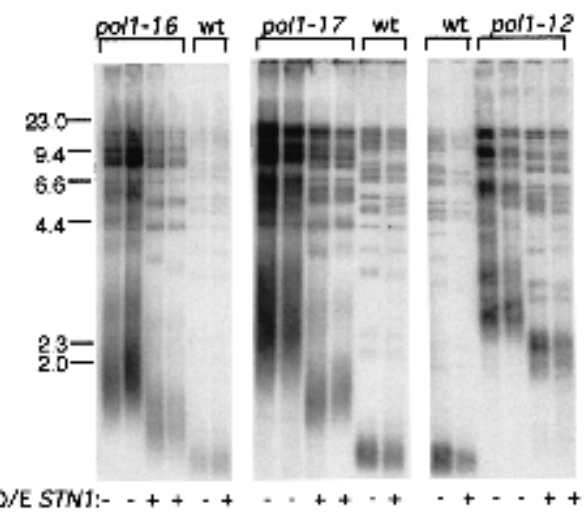

D
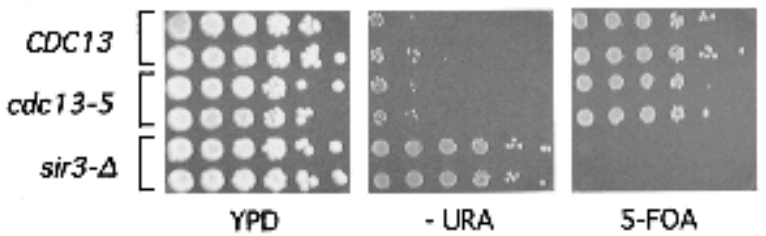

Figure 3. Overexpression of STN1 suppresses the telomere elongation phenotypes of $c d c 13-5$ and pol1 mutant strains. $(A)$ Telomere length of a $c d c 13-\Delta$ strain covered by a $C D C 13^{+}$plasmid (pVL440; lanes 1-4) or a $c d c 13-5$ plasmid (pVL1033; lanes 5-8) plus a plasmid overexpressing STN1 (pVL1035) or a control vector (pVL1036). (B) DNA prepared from a cdc13- $\Delta$ strain covered by a CDC13 ${ }^{+}$plasmid (pVL440; lanes 3,4) or a $c d c 13-5$ plasmid (pVL1033; lanes 5,6) plus a plasmid overexpressing STN1 (pVL1035; lanes 4,6) or a control vector (pVL1036; lanes 3,5) was analyzed by nondenaturing in-gel hybridization with a $\mathrm{C}_{1-3} \mathrm{~A}$ oligonucleotide probe (left); denatured and rehybridized with a $\mathrm{Y}^{\prime}$ probe (middle); and denatured and rehybridized with a $\mathrm{C}_{1-3} \mathrm{~A}^{\prime}$ oligonucleotide probe (right); lanes 1,2: $\mathrm{pVL} 260$ heat denatured or nondenatured, respectively; lane 7: pJH354, containing a region of $\mathrm{Y}^{\prime}$ sequence. $(C)$ Telomere length of the indicated pol1 mutant strains or the isogenic POL1+ strain (indicated as wt) transformed with either a plasmid overexpressing STN1 (pVL1034) or vector (YEplac112). (D) CDC13 (UCC3505, Singer and Gottschling 1994), cdc13-5 (TVL422) and sir3- $\Delta$ (TV318), each containing a telomere-proximal URA3 gene, were grown in rich media, and 10-fold serial dilutions were plated on rich media, on media lacking uracil, and on media containing 5-FOA and incubated for $48 \mathrm{~h}$ at $30^{\circ} \mathrm{C}$. 
lagging strand synthesis (Diede and Gottschling 1999; Adams Martin et al. 2000). Alterations in telomeric chromatin are often assessed by monitoring the metastable repression of transcription of reporter genes placed adjacent to the telomeric $\mathrm{G}_{1-3} \mathrm{~T}$ telomeric tract, referred to as telomeric silencing (Gottschling et al. 1990). In a pol117 mutant strain, telomeric silencing is greatly reduced, even under growth conditions where telomeres have not yet undergone extensive elongation (Adams Martin et al. 2000). In contrast, telomeric silencing is unaltered in the cdc13-5 strain (Fig. 3D), indicating that the structure of duplex telomeric chromatin is unaffected in this mutant. This suggests that the telomere elongation observed in the cdc13-5 mutant strain is a consequence of events that are restricted to the very terminus.

\section{Stn1 interaction with Cdc13 is abolished} by the cdc13-2 mutation

Increased expression of STN1 suppressed the telomere length phenotypes of the $c d c 13-5$ strain (Fig. 3) but did not suppress the $c d c 13-2$ defect; in fact, the senescence phenotype of a $c d c 13-2$ strain was enhanced when the negative regulator STN1 was overexpressed (data not shown). In contrast, the $c d c 13-2$ phenotype is partially suppressed when the positive regulator EST1 is overexpressed (Nugent et al. 1996), which is also consistent with recent work indicating that Est1 binds to a site on Cdc13 that is abolished by the cdc13-2 mutation (Pennock et al., 2001). Since two-hybrid analysis had previously shown that Stn 1 and Cdc13 also interact (Grandin et al. 1997), we tested whether Stn1 performs its negative regulatory role by binding to a similar domain on $\mathrm{Cdc} 13$ as is bound by Est1. Figure 4 shows that $\operatorname{Stn} 1$ and Cdc13 exhibit a strong two-hybrid interaction, as previously observed. Strikingly, the $c d c 13-2$ mutation abrogates this Stn1-Cdc13 interaction. This effect is specific to this mutation, as association between Stn 1 and Cdc13 is still observed when either the $c d c 13-1$ or $c d c 13-3$ mutations are introduced (Fig. 4; data not shown).

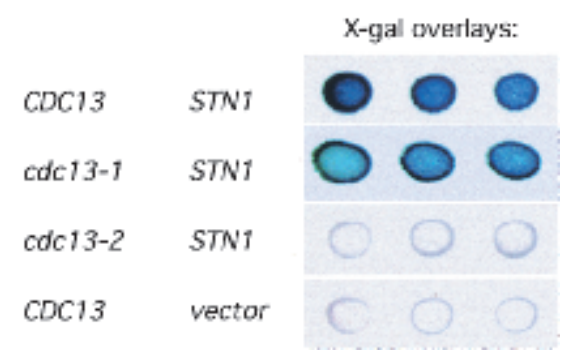

Figure 4. Cdc13, but not Cdc13-2, interacts with Stn1, by a two-hybrid test. Activation of a lacZ two-hybrid reporter gene was evaluated using X-gal overlay assays to detect $\beta$-galactosidase activity. Results are shown for three independent transformants each of CDC13 and STN1 (pVL705 and pVL859), cdc13-1 and STN1 (pVL667 and pVL859), cdc13-2 and STN1 (pVL854 and pVL859), and CDC13 and vector (pVL705 and pACT2). The ADE2 and HIS3 reporter genes present in this strain (pJ69-4A; James et al. 1996) gave results similar to the lacZ reporter (data not shown).
The increased single-stranded telomeric DNA present in a cdc13-5 strain does not invoke a DNA damage response

Cdc13 has an essential function in protecting the chromosome termini from degradation, as loss of $C D C 13$ function (using the thermolabile $c d c 13-1$ allele characterized previously) results in substantial resection of the $\mathrm{C}$ strand of the telomere and a resulting $R A D$ 9-mediated $\mathrm{G}_{2} / \mathrm{M}$ arrest (Weinert and Hartwell 1993; Garvik et al. 1995; Diede and Gottschling 1999). This indicates that Cdc13 has an essential function in protecting the chromosome termini from degradation (Garvik et al. 1995; Nugent et al. 1996). The cdc13-5 strain, in contrast, does not appear to be defective for this essential function of $C D C 13$ because it does not exhibit either growth defects or a DNA damage response. The growth of a $c d c 13-5$ strain was comparable to that of a wild-type strain at both $30^{\circ} \mathrm{C}$ and $36^{\circ} \mathrm{C}$, temperatures that are not permissive for growth of the thermolabile $c d c 13-1$ strain (Fig. 5A). Both FACS data (Fig. 2B) and bud index analysis (data not shown) showed that the $c d c 13-5$ strain exhibited the same cell cycle distribution as a wild-type strain at $30^{\circ} \mathrm{C}$. In addition, the kinetics of microcolony formation for a $c d c 13-5$ strain was comparable to that of wild type, whereas a $c d c 13-1$ strain arrested as large budded cells at $30^{\circ} \mathrm{C}$ (Fig. 5B). Thus, the $c d c 13-5$ mutant does not show a noticeable growth defect or an alteration in cell cycle progression, showing that the activity of $C D C 13$ that is responsible for negative regulation of telomere length is distinct from the essential end protection function of CDC13. Furthermore, expression of the RNR genes, which are normally induced in response to DNA damage or replication blocks (Elledge et al. 1993), was comparable in the $c d c 13-5$ and CDC13 strains, in contrast to the robust induction of $R N R$ expression that was observed either in a $c d c 13-1$ strain at a nonpermissive temperature $\left(30^{\circ} \mathrm{C}\right)$ or in a $C D C 13$ strain treated with $\mathrm{HU}$ (Fig. 5C). In addition, genome-wide expression analysis of a $c d c 13-3$ strain did not reveal any characteristic expression patterns suggesting a response to DNA damage, clearly distinguishing it from $c d c 13-1$ (T.R. Hughes et al., in prep.). Therefore, the substantial increase in the amount of single-stranded DNA at the telomere that is observed in $c d c 13-3$ and $c d c 13-5$ strains is not sufficient to invoke a DNA damage response.

This suggests that the DNA damage response of the cdc13-1 mutant strain may be the combined consequence of the simultaneous increase in single-stranded DNA and the loss of the unstable Cdc13-1 protein from chromosome ends. The lack of a DNA damage response evoked by the $c d c 13-5$ mutant could be explained if the extended single-stranded G tails are transient and/or are still bound (and hence protected) by the Cdc13 protein. The latter suggestion is also compatible with the previous demonstration that a single-stranded telomeric oligomer is capable of binding multiple Cdc 13 molecules (Hughes et al. 2000a). Consistent with such a model, the binding affinity of the truncated $80-\mathrm{kD}$ version of the Cdc13-3 protein to a 24-base oligonucleotide was almost 


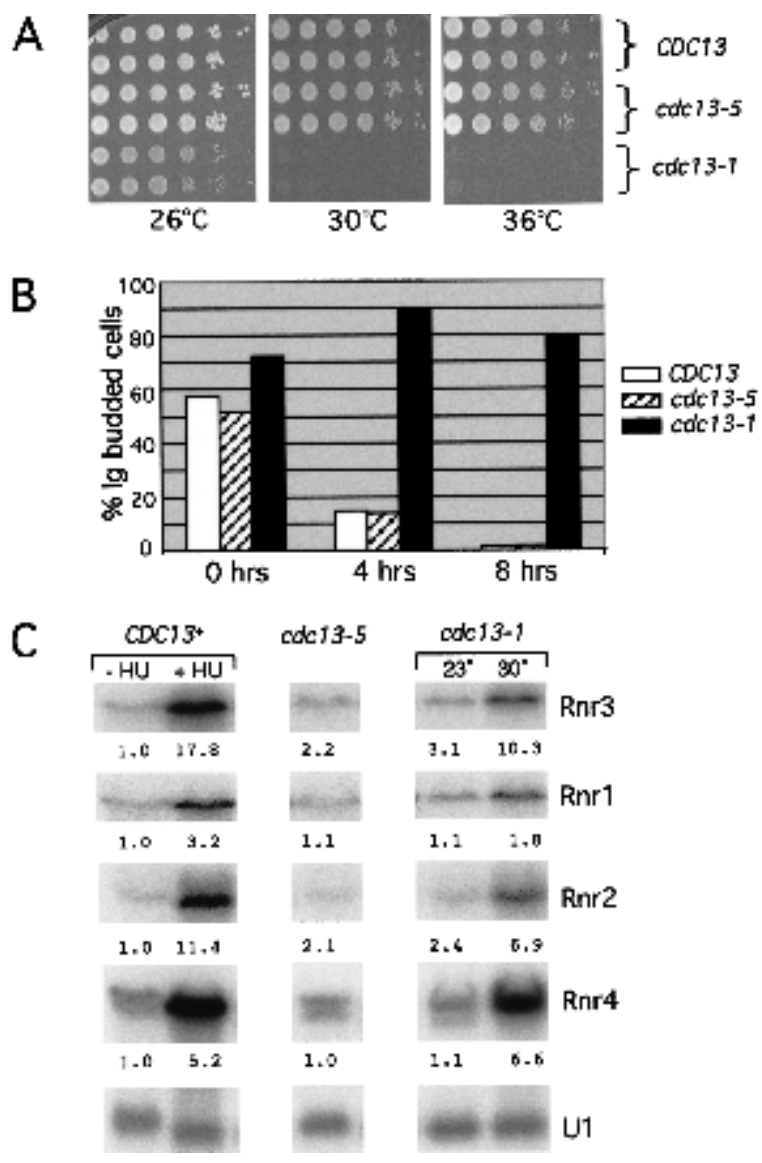

Figure 5. The $c d c 13-5$ mutation is not compromised for the essential role of $C D C 13$. (A) 10 -fold serial dilutions of $C D C 13^{+}$, cdc13-5, and $c d c 13-1$ strains were plated on rich media and incubated at $26^{\circ} \mathrm{C}, 30^{\circ} \mathrm{C}$, and $36^{\circ} \mathrm{C}$. $(B)$ Progression of individual cells to microcolonies, for $C D C 13, c d c 13-1$, and $c d c 13-5$ strains, measured as described by Weinert and Hartwell 1993. Strains were grown to early $\log$ growth phase at $23^{\circ} \mathrm{C}$ and plated for individual cells on prewarmed plates at $30^{\circ} \mathrm{C}$ (a nonpermissive temperature for the $c d c 13-1$ strain); for each genotype, $\geq 100$ cells were monitored at 0,4 , and $8 \mathrm{~h}$ for the proportion of large budded cells. $(C)$ Northern analysis monitoring the expression level of $R N R$ genes. $C D C 13^{+}$and $c d c 13-5$ strains were grown to an $\mathrm{OD}_{600}$ of 0.4 at $30^{\circ} \mathrm{C}$, and an aliquot of $C D C 13^{+}$cells was incubated in the presence of $200 \mathrm{mM}$ hydroxyurea for $3 \mathrm{~h}$; the cdc13-1 strain was grown at $23^{\circ} \mathrm{C}$ and an aliquot shifted to $30^{\circ} \mathrm{C}$ for $3 \mathrm{~h}$. Relative $R N R$ transcript levels are shown, normalized to the fold difference between the level of the $R N R$ transcript relative to the U1 transcript levels in the wild-type strain.

identical to that of wild-type Cdc13 protein (Fig. 6; data not shown). We therefore propose that the extended single-stranded DNA present at telomeres in cdc13-3 and $c d c 13-5$ strains is still bound by $\mathrm{Cdc} 13$ (and presumably other factors) and is incapable of sending a DNA damage signal.

\section{Discussion}

Several previous studies, in both ciliates and budding yeast, indicate that coordinated synthesis of the two strands of the telomere is required for telomere length maintenance. Disruption of the activity of the lagging strand DNA replication machinery, by either mutation or pharmacological intervention, leads to unregulated elongation of the G-rich strand, accompanied by an impaired inability to regulate synthesis of the C-rich strand. However, the specific mechanism that coordinates G-strand synthesis by telomerase with C-strand synthesis by the lagging strand synthesis machinery has not been elucidated.

The work presented here shows that $\mathrm{Cdc} 13$ negatively regulates elongation of the $\mathrm{G}$ strand and that this activity is a key component in the coordination between Gstrand and C-strand synthesis. A model to explain this negative regulatory role must also take into account the fact that $\mathrm{Cdc} 13$ positively regulates telomere replication as well. One simple proposal, presented in Figure 7, is derived from a model presented by Fan and Price (1997), based on their studies of de novo telomere replication in the ciliate Euplotes crassus. These authors proposed that following elongation of the G strand by telomerase, fillin synthesis of the $\mathrm{C}$ strand could limit further addition of telomeric repeats, possibly by displacement or inhibition of telomerase by the pol $\alpha /$ primase complex. In Figure 7, we expand their model by proposing that the two regulatory activities of $\mathrm{Cdc} 13$, defined by the $c d c 13-2$ and $c d c 13-5$ defects, correspond to two distinct steps in telomere replication that coordinate and regulate synthesis of the two strands of the telomere. Binding of Cdc13 to the transiently exposed single-strand G tails present in S phase initiates telomere replication by recruiting telomerase, which can then extend the $3^{\prime}$ terminus of the G-rich strand. Subsequent fill-in synthesis of the $\mathrm{C}$ strand generates a terminus that no longer exhibits an extended single-stranded end structure. We propose that synthesis of the $\mathrm{C}$ strand by lagging strand synthesis machinery acts to limit extension of the G strand by telomerase, and it is this inhibitory second

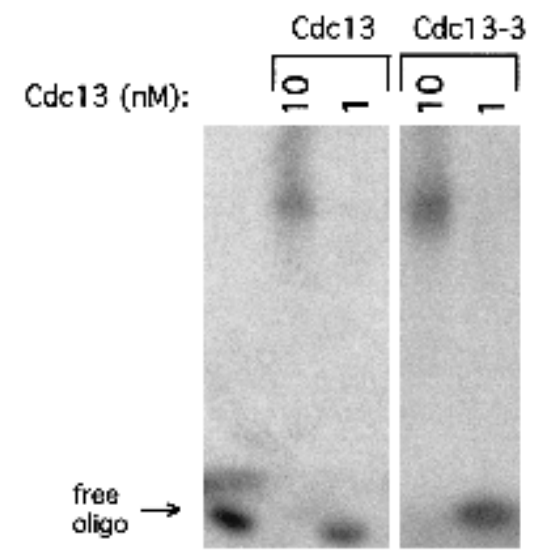

Figure 6. The $80-\mathrm{kD}$ truncated Cdc13 protein retains DNA binding activity. DNA binding ability of wild-type Cdc13 (lanes 2,3 ) and the $80-\mathrm{kD}$ truncated derivative of Cdc13-3 (lanes 4,5), as assessed by DNA gel shift assays with 5 ' end-labeled d(TGT GTGGG) $)_{3}$ oligomer at $1 \mathrm{nM}$ and protein concentration at 10 or $1 \mathrm{nM}$, as indicated; lane 1, no protein added. 
in $\mathrm{CDCl}^{3}$ :

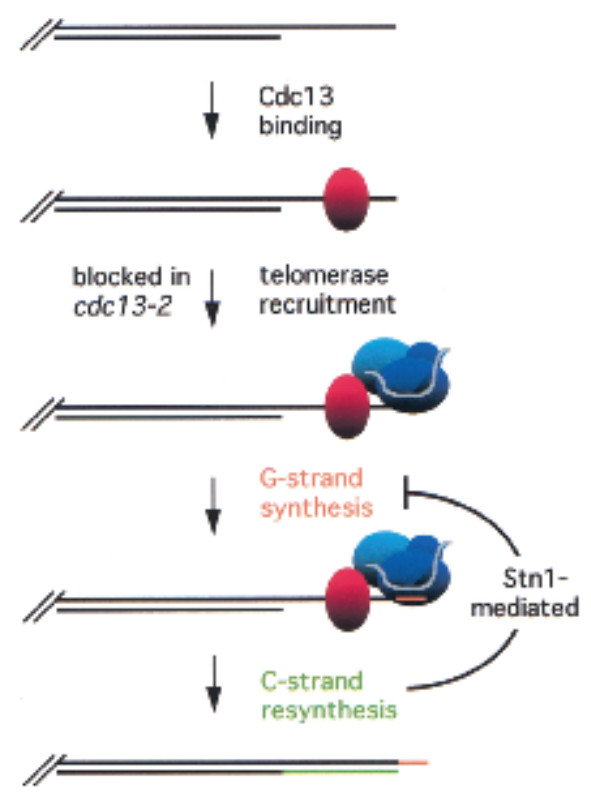

in codc 13-5:

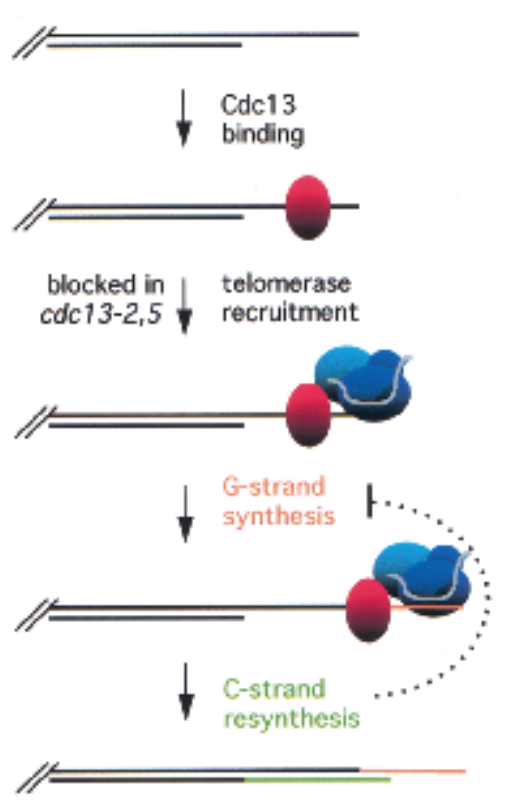

Figure 7. Cdc13 plays a dual role in regulation of G-strand synthesis by telomerase. The extended G strand, which is generated by a telomerase-independent mechanism (Wellinger et al. 1996), provides a substrate for binding by Cdc13. Once bound to the telomere, Cdc13 initiates synthesis of the G strand by recruiting telomerase through an interaction with the Estl telomerase subunit. Binding of Est1 to Cdc13, and hence enzyme recruitment, is blocked by the $c d c 13-2$ mutation. Elongation of the G strand by telomerase is subsequently followed by fill-in synthesis of the $\mathrm{C}$ strand by lagging strand synthesis machinery, which inhibits further synthesis of the G strand by telomerase. This inhibitory step is defective in the cdc13-5 mutant, allowing telomerase to further extend the G strand. We propose that binding of Stn1 to Cdc13, thereby displacing telomerase, is the mechanism by which unchecked telomerase synthesis is inhibited. regulatory step that is defective in the cdc13-5 mutant. Thus, the first step (telomerase recruitment) occurs normally in the $c d c 13-5$ strain, but the subsequent response to the initiation of C-strand DNA replication is impaired or delayed, allowing telomerase to continue unchecked in its synthesis of the G strand.

This model is supported by several observations. First, the telomere elongation defect of the cdc13-5 mutant is dependent on an intact telomerase complex, as well as the recruitment activity of Cdc13. In addition, the enhanced single-strand extension of the G strand observed in the cdc13-5 mutant is also restricted to the same period of the cell cycle when telomeres are normally elongated (Marcand et al. 2000). Two experiments indicate that regulation of C-strand synthesis is also an important component of the phenotype of the $c d c 13-5$ mutant. The telomere length defects of both $c d c 13-5$ and pol1 mutant strains are suppressed by overexpression of the Stn 1 protein, indicating that Stn 1 helps coordinate the action of the lagging strand replication complex with the regulatory activity of CDC13. Furthermore, the cdc13-5 strain displays an extreme enhancement of the telomere defect in response to altered dosage of one subunit of the lagging strand DNA replication machinery, arguing that the cdc13-5 defect is correlated with the ability to synthesize the C strand (data not shown). Finally, the lack of a silencing defect in the cdc13-5 strain argues that the telomere elongation phenotype is not a secondary consequence of a more general disruption of duplex telomeric chromatin structure.

Several lines of evidence indicate that both Stn 1 and Est1 bind to the Cdc13 protein. Therefore, we suggest that the two steps in regulation of G-strand synthesis may be the consequence of the binding of two successive complexes to Cdc13. Work presented elsewhere demonstrates that the telomerase-associated Est1 protein pro- motes the positive regulatory step in telomere replication by binding to the recruitment domain of Cdc13, an interaction that is eliminated by the $c d c 13-2$ mutation (Nugent et al. 1996; Qi and Zakian 2000; Pennock et al., 2001). In this work, we show that interaction of the negative regulator Stn1 with Cdc13 is also abolished by the same mutation. Thus, binding of Stn 1 to Cdc13, occurring in response to C-strand synthesis, could limit the extent of G-strand synthesis by displacing telomerase from Cdc13. This proposed mechanism predicts that Est 1 and Stn 1 should compete for binding to overlapping binding sites on Cdc13 (defined by the $c d c 13-2$ missense mutation), a possibility that is currently under investigation.

The $c d c 13-5$ mutation, which is caused by the loss of the 230 terminal amino acids of Cdc13, still retains the site defined by the $c d c 13-2$ mutation. Based on the above model, loss of the C-terminal region presumably impairs the ability of the Cdc13-5 protein to interact with Stn1. This is consistent with rescue of the $c d c 13-5$ defect by overexpression of STN1, indicating that increased Stn1 protein levels can restore an interaction between Stn 1 and the truncated Cdc13-5 protein. Therefore, the presence of the C-terminal 230 amino acids of Cdc13, although not essential for interaction with Stn1, may help stabilize or facilitate the Stn1-Cdc13 association. It is possible that the $\mathrm{C}$ terminus of $\mathrm{Cdc} 13$ interacts with yet another protein that could modulate the proposed competition for binding between Est1 and Stn1.

We have previously described another means by which the limit on telomere length homeostasis can be overcome, by fusing a subunit of telomerase to Cdc13 or to the DNA binding domain of $\mathrm{Cdc13}, \mathrm{DBD}_{\mathrm{Cdc13}}$ (Evans and Lundblad 1999; Hughes et al. 2000b). These telomerase fusions result in greatly elongated telomeres, presumably because the first step in telomere length regu- 
lation-the recruitment step-has been greatly enhanced. This contrasts with the proposal, above, that telomere elongation caused by the cdc13-5 mutation is due to a defect subsequent to the recruitment step. Comparison of the properties of these two perturbations of telomere length control supports the idea that two different regulatory steps in telomere replication are altered. First, telomere elongation by $\mathrm{DBD}_{\mathrm{Cdc} 13}$-telomerase or Cdc13-telomerase fusions is the result of a gainof-function property, whereas the cdc13-5 defect is a recessive mutation. Second, elongation by the Cdc13telomerase fusions is not dependent on the activity defined by the cdc13-2 mutation (Evans and Lundblad 1999; Hughes et al. 2000b), whereas telomerase elongation by the cdc13-5 mutation cannot occur in the presence of the recruitment-defective cdc13-2 allele. Finally, the Cdc13-5 mutant protein does not exhibit a detectable association with telomerase (S.K. Evans, A. Chandra, and V. Lundblad, unpubl.), whereas the Cdc13-Est1 and Cdc13-Est2 fusions both retain tight association with an active enzyme complex (Evans and Lundblad 1999).

One potentially surprising consequence of this study is the observation that the extensive single-stranded regions present during $S$ phase in a $c d c 13-5$ strain can be tolerated without obvious effects on cell cycle progression or genomic stability. A cdc13-5 strain does not exhibit a DNA damage response (Fig. 5), nor is chromosome loss elevated in this strain (data not shown). This contrasts with previous studies of the cdc13-1 mutant strain, which exhibits increased mitotic recombination and chromosome loss at semipermissive temperatures (Carson and Hartwell 1985; Hartwell and Smith 1985) and a severe DNA damage response and extensive loss of C-strand DNA under nonpermissive conditions (Weinert and Hartwell 1993; Garvik et al. 1995). Diede and Gottschling (1999) have proposed that tight coupling between telomerase synthesis and the activity of DNA polymerases $\alpha$ and $\delta$ is necessary to prevent inappropriately elongated G-tails, which could elicit the same detrimental consequences similar to those observed in a cdc13-1 mutant. One explanation for the behavior of the cdc13-5 strain that would still be consistent with their proposal is that the $c d c 13-5$ mutation may not be a complete coupling defect, as the elongated G tails are only observed in $S$ phase in this strain. Because telomeres appear to be primarily duplex at other stages of the cell cycle in the cdc13-5 strain, most of the C strand must be eventually synthesized during each cell cycle in this mutant. This indicates that there is a delay, rather than a complete block, in coupling between synthesis of the two strands. However, if the proposed coupling function is essential for cell cycle progression, it is notable that the cdc13-5 strain displays no apparent cell cycle delay. This suggests that tight coupling may not be essential and that the cell may be able to maintain genomic integrity even in the presence of elongated single-stranded tracts at the telomere (at least during one period of the cell cycle), as long as these single-stranded regions are bound and protected by Cdc13.

In this article, we have described a role for Cdc13 in the negative regulation of telomere length by coordinating G-strand and C-strand synthesis. This mechanism appears to be distinct from that promoted by duplex telomere DNA binding proteins, which mediate a conformational switch that affects the accessibility of the chromosome end to telomerase (for review, see Evans and Lundblad 2000). Notably, like CDC13, the mammalian telomere binding protein TRF2 is required for both negative regulation of telomere length (Smogorzewska et al. 2000) and an essential role in chromosome end protection (van Steensel et al. 1998). Both end protection and length regulation of human telomeres may rely on the regulated formation of a unique DNA structure, dubbed the t-loop, in which the G-rich 3' overhang invades the duplex telomeric tract (Griffith et al. 1999). Whether such a structure forms at yeast telomeres, and is regulated by the Cdc13 protein, is an interesting experimental question.

\section{Materials and methods}

\section{Strains and plasmids}

All strains used in this study are isogenic, derived from previously published strains by introduction of the relevant deletion mutations or $c d c 13$ mutations by standard molecular techniques, with the exception of the strains used in Figure 3C and 3D. DVL233 (MATa/ $\alpha$ ura3-52/ura3-52 ade2-101/ade2-101

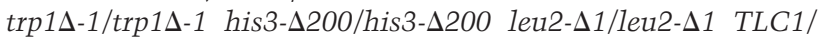
tlc1 $::$ LEU2 RAD52/rad52- $\Delta:: L E U 2 \quad$ CDC13/cdc13-3 CFSUP11-TRP1) and DVL326 (MATa/ $\alpha$ ura3-52/ura3-52 ade2-101/

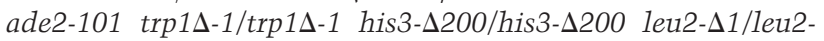
$\Delta 1$ TLC1/t1c1- $\Delta:: L E U 2$ CDC13/cdc13-5 CF-SUP11-TRP1) were derived from DVL131 as described (Lendvay et al. 1996),

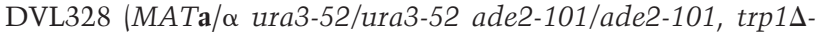

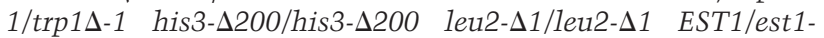
$\Delta 3:: H I S 3$ RAD52/rad52- $\Delta:: L E U 2$ CDC13/cdc13-5 CF-SUP11TRP1) was derived from TVL140 as described previously (Lundblad and Blackburn 1993), and DVL162 (MATa/ a ura3-52/ura3-

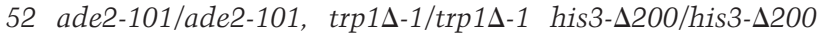

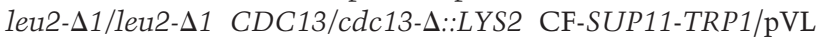
438) from YPH275 (Lundblad and Szostak 1989). Haploid cdc131, $c d c 13-3, c d c 13-5$, or $c d c 13-2,5$ strains were derived by either dissecting the relevant diploid or by introducing the relevant plasmids into a $c d c 13-\Delta /$ pVL438 haploid strain (derived from dissection of DVL162), followed by eviction of pVL438 on selective media containing 5-FOA.

All CDC13 plasmids used in this study were derived from pVL438 (CEN URA3 CDC13; YCplac33-based) or pVL440 (CEN LEU2 CDC13; YCplac111-based); both contained the same 4.5$\mathrm{kb}$ genomic insert with the wild-type CDC13 gene. pVL690 (CEN LEU2 cdc13-2), pVL762 (CEN LEU2 cdc13-1), and pVL1360 (CEN LEU2 cdc13-2,5) were derived from pVL440 by introducing the relevant CDC13 mutant alleles. The cdc13-3 mutation (K695A, R697A, D698A, E699A) was constructed by site-directed mutagenesis in the E. coli expression plasmid pVL427 (Hughes et al. 2000a) and subsequently subcloned to generate pVL821 (CEN LEU2 cdc13-3). The cdc13-5 mutation was constructed by PCR to introduce a stop codon following amino acid 694 to generate pVL1033 (CEN LEU2 cdc13-5). The cdc13-3 and $c d c 13-5$ mutations were integrated into the genome of appropriate strains using pVL780 (YIp URA3 cdc13-3) or pVL1215 (YIp URA3 cdc13-5), using a pop-in/pop-out strategy. pVL841 and pVL903, which encode HA3-Cdc13p and 
HA3-Cdc13-5p, respectively, are derived from pVL440; the HA3 epitope tag introduced into the $\mathrm{N}$ terminus of $\mathrm{Cdc} 13$ was described previously (pVL842; Hughes et al. 2000b).

Plasmids used for high copy expression of STN1 were derived from YEplac112. pVL1034 ( $2 \mu$ TRP1 STN1, with expression of STN1 driven by its native promoter) contains a $2.9-\mathrm{kb}$ genomic insert that includes the STN1 gene. pVL1035 (2 $\mu$ TRP1 GALSTN1) was derived from pVL1036 (YEplac112 containing the $\sim 0.8$-kb GAL1 promoter) by insertion of a PCR-amplified copy of STN1 behind the GAL1 promoter. The plasmids used for two-hybrid analysis were derived from pAS1 and pACT1. pVL705 was constructed by insertion of a full-length fragment of CDC13 bearing an in-frame deletion of amino acids 585-677 (within the DNA binding domain of Cdc13; Hughes et al. 2000a) into the NdeI site of pAS1 to generate a fusion to the Gal4 DNA-binding domain; deletion of a portion of the DNA-binding domain was necessary to prevent overexpression lethality (C. Nugent, unpubl.). pVL854 is identical to pVL705 except for the presence of the $c d c 13-2$ missense mutation. pVL667 contains the full-length $c d c 13-1$ gene inserted into pAS1. pVL859 was recovered from a library screen as a Cdc13-interacting clone and contains amino acids 5-495 of the Stn1 protein fused in frame with the Gal4 activation domain of pACT1. pVL260 contains $80 \mathrm{bp}$ of $\mathrm{G}_{1-3} \mathrm{~T}$ DNA cloned into the polylinker of pDS67 (a pBluescript plasmid that also contains $A R G 4)$.

\section{Molecular methods}

Standard denaturing Southern gel conditions were used for determining telomere length (Lendvay et al. 1996). Single-strand chromosome termini were analyzed by nondenaturing in-gel hybridization with a 22-mer CA-rich oligonucleotide as described previously (Dionne and Wellinger 1996). To assay chromosome end structure during the cell cycle, the $c d c 13-5$ strain was grown in YPD to $\mathrm{OD}_{600} 0.2$, followed by the addition of 3 $\mu \mathrm{M} \alpha$ factor. Additional $\alpha$ factor (at a concentration of $3 \mu \mathrm{M}$ ) was added every hour until $~ 90 \%$ of the cells appeared unbudded (for $\sim 3-4 \mathrm{~h})$. The $\alpha$ factor was removed by centrifugation and cells released into YPD at $\mathrm{OD}_{600}$ 0.4. Cells for FACS analysis were fixed in $70 \%$ ethanol, washed in $50 \mathrm{mM} \mathrm{Na}$ citrate $(\mathrm{pH} 7.0$ ), digested with RNase overnight, washed again and stained with propidium iodide $(15 \mu \mathrm{g} / \mathrm{mL})$, and analyzed by flow cytometry.

For Northern blot analysis, total RNA was isolated by a phenol-freeze method (Schmitt et al. 1990), resolved on formaldehyde- $1 \%$ agarose, and probed with the 1.7-kb BgIII-EcoRI RNR1 fragment from pSE738, the 1.1-kb HindIII RNR2 fragment from pSE310, the 1-kb BamHI-EcoRI RNR3 fragment from pSE734, the 1.4-kb StuI-SwaI RNR4 fragment from pMH120, or a 600-bp fragment of the $U 1$ gene (recovered by PCR of yeast genomic DNA).

\section{Biochemical methods}

For detection of the HA-tagged $\mathrm{Cdc} 13$ and $\mathrm{Cdc} 13-5$ proteins, $c d c 13-\Delta /$ pVL440 (Cdc13), $c d c 13-\Delta /$ pVL841 (HA3-Cdc13), and $c d c 13-\Delta /$ pVL903 (HA3-Cdc13-5) were grown in selective media to an $\mathrm{OD}_{600}$ 0.6. Extract preparation and Western analyses were done as described in Hughes et al. (2000b), except that TMG $300+\left(10 \mathrm{mM}\right.$ Tris- $\mathrm{HCl}$ at $\mathrm{pH} 8.0,1 \mathrm{mM} \mathrm{MgCl}_{2}, 5 \%$ glycerol, 1 mM PMSF, $1 \mathrm{mM} \mathrm{DTT,} 300 \mathrm{mM} \mathrm{NaCl}$ ) was used for immunoprecipitations and washes.

His $_{6}$-tagged proteins (Cdc13 or Cdc13-3) were expressed in SF9 cells as described previously (Hughes et al. 2000a). For protein production, cells were pelleted, washed, and resuspended in SB $30010 \%+(50 \mathrm{mM}$ Na phosphate at pH 8.0, $300 \mathrm{mM} \mathrm{NaCl}$, $10 \%$ glycerol, $0.5 \%$ Tween-20, 10 mM imidazole, 1 mM PMSF), and sonicated for 3-4 repetitions of 10-30 sec; the degree of sonication required was monitored by visual inspection. Extracts were clarified by centrifugation for $10 \mathrm{~min}$, and His $_{6}$-tagged proteins were purified on Ni-NTA agarose at $4^{\circ}$ (QIAGEN) by batch method, according to the manufacturer's instructions. Protein concentration was determined by comparison to BSA standards on Coomassie-stained SDS-PAGE gels. Binding reactions were performed as described in Nugent et al. (1996).

\section{Acknowledgments}

We thank the members of the Lundblad laboratory for many helpful discussions and also thank the Flow Cytometry Core Lab, Texas Children's Hospital. We gratefully acknowledge the generous gifts of strains or plasmids from Connie Holm, Oscar Aparicio, Stephen Bell, Judith Campbell, Steve Elledge, Tim Formosa, and Peter Burgers. This work was supported by NIH grant GM55867 (to V.L.) and a postdoctoral fellowship from the U.S. Army and Materiel Command (to C.N.).

The publication costs of this article were defrayed in part by payment of page charges. This article must therefore be hereby marked "advertisement" in accordance with 18 USC section 1734 solely to indicate this fact.

\section{References}

Adams, A.K. and Holm, C. 1996. Specific DNA replication mutations affect telomere length in Saccharomyces cerevisiae. Mol. Cell. Biol. 16: 4614-4620.

Adams Martin, A., Dionne, I., Wellinger, R.J., and Holm, C. 2000. The function of DNA polymerase $\alpha$ at telomeric G tails is important for telomere homeostasis. Mol. Cell. Biol. 20: 786-796.

Artandi, S.E., Chang, S., Lee, S.L., Alson, S., Gottlieb, G.J., Chin, L., and DePinho, R.A. 2000. Telomere dysfunction promotes non-reciprocal translocations and epithelial cancers in mice. Nature 406: 641-645.

Bodnar, A.G., Ouellette, M., Frolkis, M., Holt, S.E., Chiu, C.-P., Morin, G.B., Harley, C.B., Shay, J.W., Lichtsteiner, S., and Wright, W.E. 1998. Extension of life-span by introduction of telomerase into normal human cells. Science 279: 349-352.

Carson, M. and Hartwell, L. 1985. CDC17: An essential gene that prevents telomere elongation in yeast. Cell 42: 249-257.

Chin, L., Artandi, S.E., Shen, Q., Tam, A., Lee, S.L., Gottlieb, G.J., Greider, C.W., and DePinho, R.A. 1999. p53 deficiency rescues the adverse effects of telomere loss and cooperates with telomere dysfunction to accelerate carcinogenesis. Cell 97: 527-538.

Diede, S.J. and Gottschling, D.E. 1999. Telomerase-mediated telomere addition in vivo requires DNA primase and DNA polymerases $\alpha$ and $\beta$. Cell 99: 723-733.

Dionne, I. and Wellinger, R.J. 1996. Cell cycle-regulated generation of single-stranded G-rich DNA in the absence of telomerase. Proc. Natl. Acad. Sci. 93: 13902-13907.

Elledge, S.J., Zhou, Z., Allen, J.B., and Navas, T.A. 1993. DNA damage and cell cycle regulation of ribonucleotide reductase. Bioessays 15: 333-339.

Evans, S.K. and Lundblad, V. 1999. Est1 and Cdc13 as comediators of telomerase access. Science 286: 117-120.

- 2000. Positive and negative regulation of telomerase access to the telomere. J. Cell Sci. 113: 3357-3364.

Fan, X. and Price, C.M. 1997. Coordinate regulation of G- and C-strand length during new telomere synthesis. Mol. Biol. Cell 8: 2145-2155. 
Garvik, B., Carson, M., and Hartwell, L. 1995. Single-stranded DNA arising at telomeres in $c d c 13$ mutants may constitute a specific signal for the RAD9 checkpoint. Mol. Cell. Biol. 15: 6128-6138.

Gottschling, D.E., Aparicio, O.M., Billington, B.L., and Zakian, V.A. 1990. Position effect at S. cerevisiae telomeres: Reversible repression of Pol II transcription. Cell 63: 751-762.

Grandin, N., Reed, S.I., and Charbonneau, M. 1997. Stn1, a new Saccharomyces cerevisiae protein, is implicated in telomere size regulation in association with Cdc13. Genes \& Dev. 11: $512-527$.

Grandin, N., Damon, C., and Charbonneau, M. 2000. Cdc13 cooperates with the yeast $\mathrm{Ku}$ proteins and $\mathrm{Stn} 1$ to regulate telomerase recruitment. Mol. Cell. Biol. 20: 8397-8408.

Gravel, S., Larrivee, M., Labrecque, P., and Wellinger, R.J. 1998. Yeast $\mathrm{Ku}$ as a regulator of chromosomal DNA end structure. Science 280: 741-745.

Greider, C.W. 1996. Telomere length regulation. Ann. Rev. Biochem. 65: 337-365.

Griffith, J.D., Comeau, L., Rosenfield, S., Stansel, R.M., Bianchi, A., Moss, H., and de Lange, T. 1999. Mammalian telomeres end in a large duplex loop. Cell 97: 503-514.

Hartwell, L. and Smith, D. 1985. Altered fidelity of mitotic chromosome transmission in cell cycle mutants of S. cerevisiae. Genetics 110: 381-395.

Hughes, T.R. 1998. "Biochemical characterization of telomere replication proteins in Saccharomyces cerevisiae." Ph.D. thesis, Baylor College of Medicine.

Hughes, T.R., Weilbaecher, R.G., Walterscheid, M., and Lundblad, V. 2000a. Identification of the single-strand telomeric DNA binding domain of the Saccharomyces cerevisiae Cdc13 protein. Proc. Nat1. Acad. Sci. 97: 6457-6462.

Hughes, T.R., Evans, S.K., Weilbaecher, R.G., and Lundblad, V. $2000 \mathrm{~b}$. The Est 3 protein is a subunit of yeast telomerase. Curr. Biol. 10: 809-812.

James, P., Halladay, J., and Craig, E.A. 1996. Genomic libraries and a host strain designed for highly efficient two-hybrid selection in yeast. Genetics 144: 1425-1436.

Lee, H.W., Blasco, M.A., Gottlieb, G.J., Horner II, J.W., Greider, C.W., and DePinho, R.A. 1998. Essential role of mouse telomerase in highly proliferative organs. Nature 392: 569574 .

Lendvay, T.S., Morris, D.K., Sah, J., Balasubramanian, B., and Lundblad, V. 1996. Senescence mutants of Saccharomyces cerevisiae with a defect in telomere replication identify three additional EST genes. Genetics 144: 1399-1412.

Lin, J.J. and Zakian, V.A. 1996. The Saccharomyces CDC13 protein is a single-strand $\mathrm{TG}_{1-3}$ telomeric DNA-binding protein in vitro that affects telomere behavior in vivo. Proc. Natl. Acad. Sci. 93: 13760-13765.

Lingner, J., Cooper, J.P., and Cech, T.R. 1995. Telomerase and DNA end replication: No longer a lagging strand problem? Science 269: 1533-1534.

Lingner, J., Cech, T.R., Hughes, T.R., and Lundblad, V. 1997. Three ever shorter telomere (EST) genes are dispensable for in vitro yeast telomerase activity. Proc. Nat1. Acad. Sci. 94: 11190-11195.

Lundblad, V. and Blackburn, E.H. 1993. An alternative pathway for yeast telomere maintenance rescues est $1^{-}$senescence. Cell 73: 347-360.

Lundblad, V. and Szostak, J.W. 1989. A mutant with a defect in telomere elongation leads to senescence in yeast. Cell 57: 633-643.

Marcand, S., Brevet, V., Mann, C., and Gilson, E. 2000. Cell cycle restriction of telomere elongation. Curr. Biol. 10: 487490.
Nugent, C.I., Hughes, T.R., Lue, N.F., and Lundblad, V. 1996 Cdc13p: A single-strand telomeric DNA-binding protein with a dual role in yeast telomere maintenance. Science 274: 249-252.

Pennock, E., Buckley, K., and Lundblad, V. 2001. Cdc13 delivers separate complexes to the telomere for end protection and replication. Cell 104: 387-396.

Polotnianka, R.M., Li, J., and Lustig, A.J. 1998. The yeast $\mathrm{Ku}$ heterodimer is essential for protection of the telomere against nucleolytic and recombinational activities. Curr. Biol. 8: 831-834.

Price, C.M. 1997. Synthesis of the telomeric C-strand: A review. Biochemistry 62: 1216-1223.

Qi, H. and Zakian, V.A. 2000. The Saccharomyces telomerebinding protein Cdc13p interacts with both the catalytic subunit of DNA polymerase $\alpha$ and the telomerase-associated Est1 protein. Genes \& Dev. 14: 1777-1788.

Schmitt, M.E., Brown, T.A., and Trumpower, B.L. 1990. A rapid and simple method for preparation of RNA from Saccharomyces cerevisiae. Nucleic Acids Res. 18: 3091-3092.

Singer, M.S. and Gottschling, D.E. 1994. TLC1: Template RNA component of Saccharomyces cerevisiae telomerase. Science 266: 404-409.

Smogorzewska, A., van Steensel, B., Bianchi, A., Oelmann, S., Schaefer, M.R., Schnapp, G., and de Lange, T. 2000. Control of human telomere length by TRF1 and TRF2. Mol. Cell. Biol. 20: 1659-1668.

van Steensel, B., Smogorzewska, A., and de Lange, T. 1998. TRF2 protects human telomeres from end-to-end fusions. Cell 92: 401-413.

Weinert, T.A. and Hartwell, L.H. 1993. Cell cycle arrest of $c d c$ mutants and specificity of the RAD9 checkpoint. Genetics 134: 63-80.

Wellinger, R.J., Wolf, A.J., and Zakian, V.A. 1993. Saccharomyces telomeres acquire single-strand $\mathrm{TG}_{1-3}$ tails late in $\mathrm{S}$ phase. Cell 72: 51-60.

Wellinger, R.J., Ethier, K., Labrecque, P., and Zakian, V.A. 1996. Evidence for a new step in telomere maintenance. Cell 85: 423-433. 


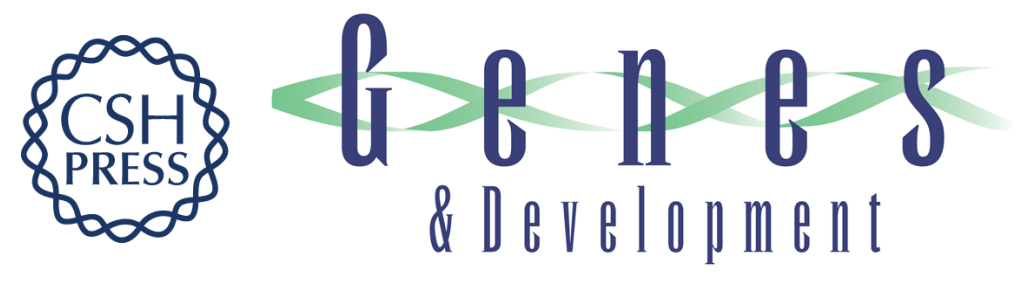

\section{Cdc13 both positively and negatively regulates telomere replication}

Abha Chandra, Timothy R. Hughes, Constance I. Nugent, et al.

Genes Dev. 2001, 15:

Access the most recent version at doi:10.1101/gad.861001

References This article cites 43 articles, 23 of which can be accessed free at: http://genesdev.cshlp.org/content/15/4/404.full.html\#ref-list-1

License

Email Alerting Receive free email alerts when new articles cite this article - sign up in the box at the top Service right corner of the article or click here.

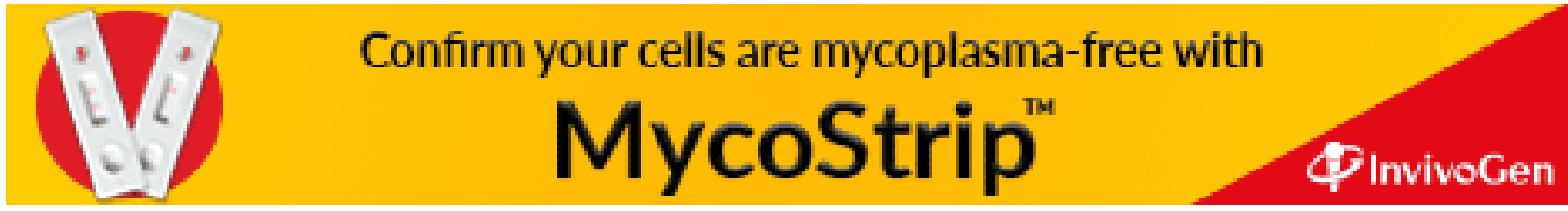

